\title{
CONTROL OF LEVITATING PARTICLE IN ULTRASOUND FIELD
}

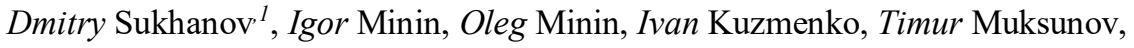 \\ Evgeniy Sivkov, Fedor Emelyanov
}

Tomsk State University, 634050, Tomsk, Russia

\begin{abstract}
The experimental setup for ultrasonic controlled levitation in the air has been developed. Two phased arrays made of 91 ultrasonic radiators placed in front of each other are used. Arrays are focused in the region of particle levitation. The length of the focus area allows us to move the particles along it, controlling the standing waves through the phase difference of the two arrays.
\end{abstract}

\section{Introduction}

Ultrasonic levitation in air for small-sized particles is ensured by the displacement of particles in the extremes of the field. Acoustic levitation in the air finds more and more applications [1-3]. In particular, the traction beam technology [1] is developed, bearings with friction minimization [2, 3]. In [4], the possibility of levitation of a large flat object (disk) with dimensions much larger than the wavelength was demonstrated. Ultrasonic levitation is also used to clean surfaces from small particles [5]. With increasing power and focusing radiation, levitation of large objects of arbitrary shape is possible [6, 7].

\section{Acoustic levitation setup}

In this paper, we propose a method for levitation of particles by focusing the field from a set of low-intensity emitters. Due to the in-phase interference of waves in a certain region, the intensity of the field is sufficient to allow the particle to levitate. To keep a particle at a certain point in space, it is necessary to create an extremum of the field in it. In this case, the particle is pushed out to the equilibrium point corresponding to the extremum of the field intensity. We consider two arrays of ultrasound sources in front of each other. The arrays consist of 91 elements placed with a certain shift to produce the focusing effect (Figure 1). We place emitters in a hexagonal grid with a step of $11 \mathrm{~mm}$ as shown in Figure 2. The phase of the emitters is selected in such a way that the field distribution at a distance of $180 \mathrm{~mm}$ is a localized maximum with diameter $20 \mathrm{~mm}$.

\footnotetext{
${ }^{1}$ Corresponding author: sdy@mail.tsu.ru
} 


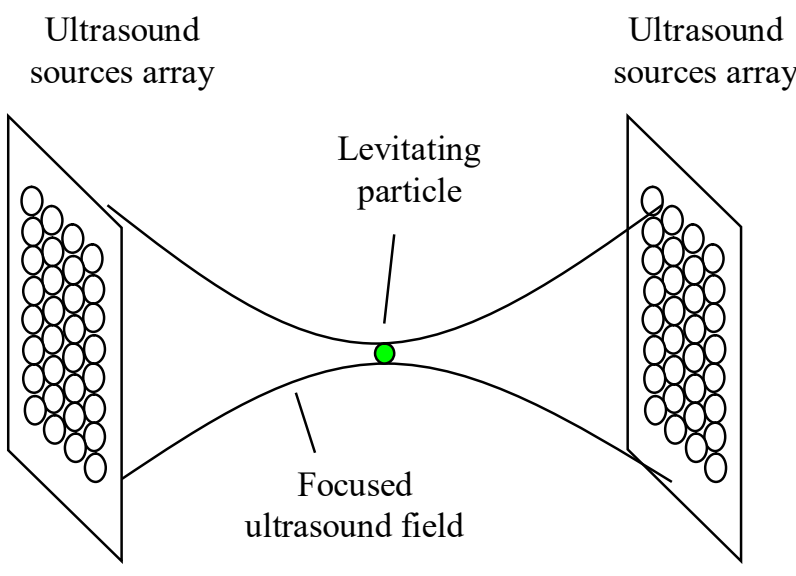

Fig.1. Ultrasonic levitation by means of phased arrays of transmitters.

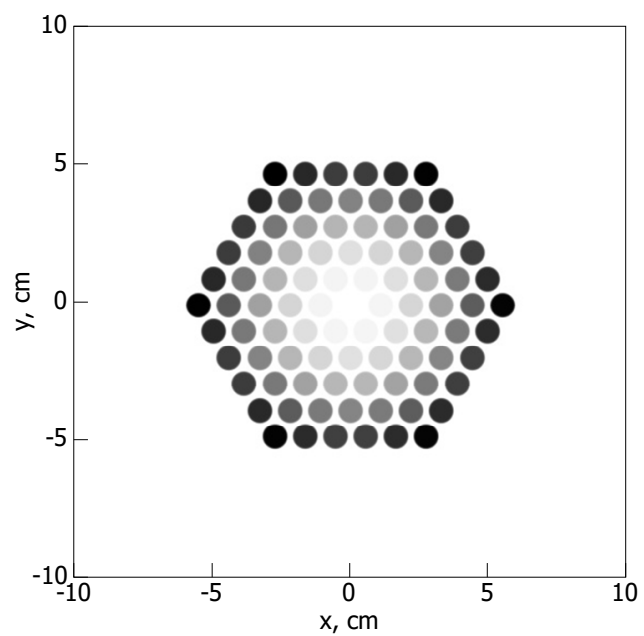

Fig. 2. Placement of source elements in array (grayscale color shows a relative depth shift of each source).

The radiation frequency is $40 \mathrm{kHz}$, which corresponds to a wavelength of $8.25 \mathrm{~mm}$ in air. To form an arbitrary field without secondary maxima, it is necessary to place the emitters at a distance of no more than $4 \mathrm{~mm}$ from each other. However, in our case, the pitch of the emitters is $11 \mathrm{~mm}$, which leads to the appearance of side maxima. Nevertheless, in our problem, the presence of lateral peaks is not a difficulty, since the levitating particle is located in the center.

For experimental studies, an array of radiators from the elements MA40S4S was created (Figure 3). All the radiators were connected in parallel to the powerful amplifier basen on the TDA7297 chip. The phase of each radiator was regulated by the height of the placement in the hole. Radiators are shifted according to a spherical surface with radius of $180 \mathrm{~cm}$. The required arrangement of 91 emitters was synthesized to form the distribution of ultrasonic waves in the form of an ultrasonic trap at a range of $180 \mathrm{~mm}$. The laboratory 
model of the installation for ultrasonic levitation in the air based on the radiator array at a frequency of $40 \mathrm{kHz}$ is shown in Figure 3.

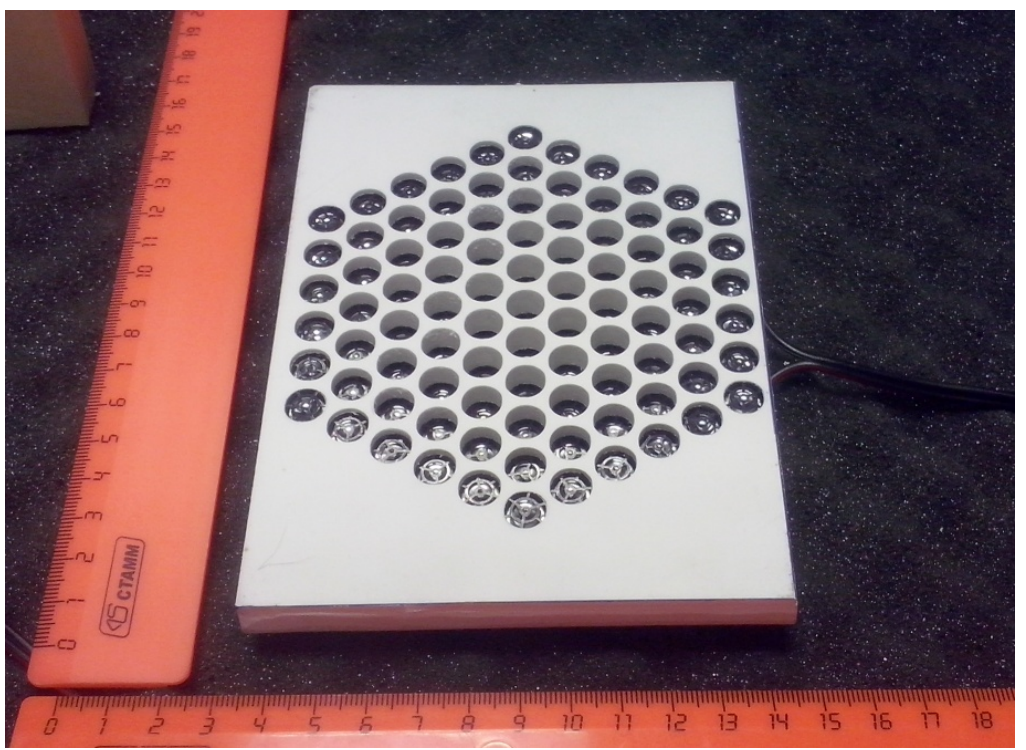

Fig. 3. Photo of sources array made of 91 elements.

To ensure the focusing properties of the source array we have made a measurements of sound field dependent on distance and horizontal shift. The resulting image of the measurement of sound field is shown on Figure 4. We can see the maximum of magnitude on distance $180 \mathrm{~mm}$. The diameter of the focusing spot is about $20 \mathrm{~mm}$.

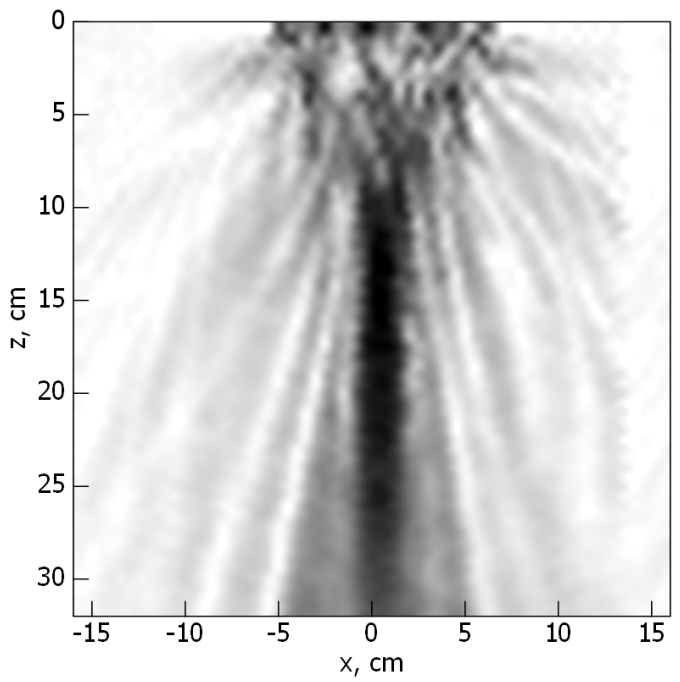

Fig.4. Measured ultrasound field magnitude.

We placed two arrays in front of each other on distance $360 \mathrm{~mm}$. Arrays was controlled by separate signals, generated by digital-analog converters with amplifiers. In the photo, in the central part, you see two white levitating particles of foam. In this case, levitation is provided in the area of focusing of both arrays. By controlling of the phase difference 
between two signals we able to shift particles horizontally. Hence the focusing area is extended by range the interval of particles shift is comparable with the half of distance between arrays. On Figure 5 you can see photos of levitating particles between two arrays.

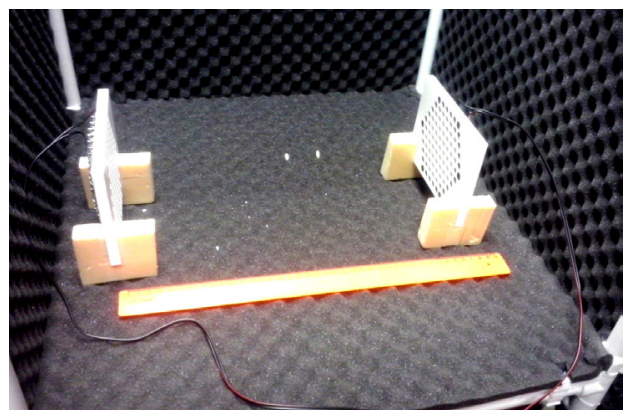

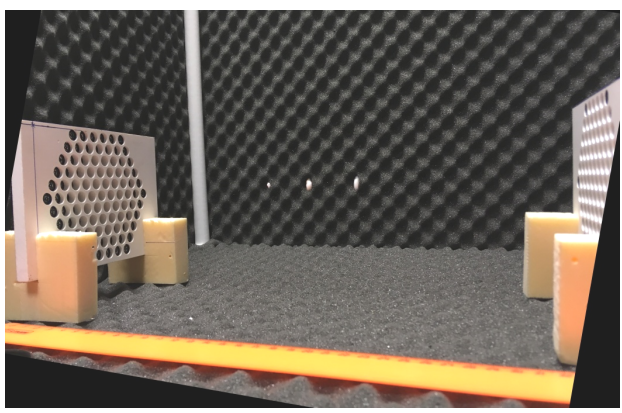

$\mathrm{b}$

Fig. 5. Photos of levitating particles: $a$ - two particles; $b$ - three particles.

Thus, the levitation of particles of polystyrene at a range of $\sim 100 \mathrm{~mm}$ near the focusing point of the radiator arrays is shown experimentally. Levitation is provided for a long time due to the influence of pressure oscillations of ultrasonic waves, as a result of which particles tend in the region of the maximum of oscillations. To shift the position of the particle, a radiator phase difference is changed, which resoling in shift of standing wave maximum.

\section{Conclusion}

Application of two radiators towards each other allows us to ensure the levitation of particles in the interference field in the focus region. The change in the height and lateral shift of the emitters led to a change in the height of the particle. Thus, the possibility of controlling the position of the particles in the range by means of adjusting the phase difference between the direct and the counter emitters is demonstrated.

\section{Acknowledgments}

The scientific research was carried out with the support of the grant of the Russian Science Foundation No. 17-79-20051.

\section{References}

[1] C.E.M. Démoré, P.M. Dahl, Z. Yang, P. Glynne-Jones, A. Melzer, S. Cochran, M. Macdonald, G.C. Spalding, Physical Review Letters 112 (17),174302 (2014)

[2] J.-S.Wang, C. Chen, G.-C. Chen, X.-J. Yan, Proc. of the 2013 Symposium on Piezoelectricity, Acoustic Waves and Device Applications, SPAWDA 2013, 6841135 (2013)

[3] C. Chen, J. Wang, B. Jia, F. Li, Journal of Intelligent Material Systems and Structures 25, 755 (2014)

[4] S. Zhao, J. Wallaschek, Archive of Applied Mechanics 81,123 (2011)

[5] A. Fuhrmann, J.S. Marshall, J. Wu, Applied Acoustics 74, 535 (2013)

[6] M.A.B. Andrade, F.T.A. Okina, A.L. Bernassau, J.C. Adamowski, Journal of the Acoustical Society of America 141, 4148 (2017)

[7] M.A.B. Andrade, A.L. Bernassau, J.C. Adamowski. Applied Physics Letters 109,044101 (2016) 